\title{
Are Singularity and Dark Energy Consequences of Vacuum Solution of Field Equations?
}

\author{
Branko M. Novakovic
}

\begin{abstract}
Modern physics is trying to solve problems like singularity, dark energy and universe expanding by using sophisticated methods in particle and quantum physics. But, the mentioned problems should also be solved in the classical General Relativity Theory (GRT). As it is well known, Schwarzschild solution of the Einstein's field equations is in fact a vacuum solution. Unfortunately, this vacuum solution generates appearances as singularity and dark energy with unknown source. Here we show that the singularity and dark energy with unknown source are direct consequence of the vacuum solution of the Einstein's field equations. In that sense we employ new Relativistic Alpha Field Theory (RAFT) that proposes solution of the field equations without appearances of singularity and offers the source of dark energy. This is the consequence of the following predictions of RAF theory: a) no a singularity in a gravitational field and $b$ ) the gravitational force becomes positive (repulsive) if $\left(G M / r c^{2}\right)>1$, that could be the source of a dark energy.
\end{abstract}

Index Terms : Relativistic alpha field theory (RAFT), Vacuum solution, Singularity, Source of dark energy

\section{INTRODUCTION}

As it is well known, vacuum solution of the Einstein's field equations predicts a singularity in a gravitational field. This solution limits the applications of GRT [1-6] to the relatively weak field, as we have in our solar system. Further, vacuum solution cannot explain what is really source of the energy that expands our universe, even in the acceleration rate. Therefore, this energy has been called dark energy, but with unknown source. While the vacuum solution of Einstein's field equations is proved to be correct in the relatively weak field in our solar system [1-6], it cannot be applied to the extremely strong field at the Planck's scale. Here we present that the new Relativistic Alpha Field Theory (RAFT) [7-10], gives the solution of the full form of the Einstein's field equations, excluding the cosmological constant $(\Lambda=0)$. This solution is without singularity and can be applied to the extremely strong fields, including of the Planck's scale. In the weak gravitational field this solution is reduced to the well-known vacuum solution of the Einstein's field equations.

One of the important discovery of the RAF theory is that gravitational force becomes positive in the region $r_{\min } \leq r<r_{c}$. Here $r_{\min }$ is the minimal radius in a gravitational field $\left(r_{\min }=\right.$ $\mathrm{GM} / 2 \mathrm{c}^{2}$ ) and $r_{\mathrm{c}}$ is the radius where negative acceleration is changes into the positive one and vice versa $\left(r_{c}=G M / c^{2}\right)$. Therefore, gravitational radius less than $r_{\min }$ is not possible and it protects from the singularity point at the zero radius at $r$ $=0$. In the region $\left(r_{c}<r<\infty\right)$ gravitational acceleration is

Branko Novakovic, FSB - University of Zagreb, Luciceva 5, P.O.B. 509, 10000 Zagreb, Croatia negative as we know from our todays knowledge. Since our universe is expanding at the acceleration rate [27-34,38], the present universe radius should be in the region $r_{\min } \leq r<r_{c}$.

It has been theoretically proved that the metrics of the line element in RAF theory is regular at the Schwarzschild radius. This means that no a singularity at the Schwarzschild radius in RAF theory. On the other side, in the vacuum solution there no natural limitation to the gravitational radius that it decrease to the singular point at zero radius. This is theoretical proof that the vacuum solution produces singularity in a gravitational field.

Further, it has been theoretically proved that at the minimal radius $r_{\text {min }}$ the all field parameters in RAF theory are regular. This means that the metrics of RAF theory is regular in the region $\mathrm{r}_{\min } \leq \mathrm{r}<\infty$. In the case that the radius is less than the minimal radius, $r<r_{\text {min }}$, the metrics in RAF theory becomes imaginary. This proves the prediction of RAF theory that there exists a minimal radius at $r=\left(G M / 2 c^{2}\right)$ that prevents singularity at $r=0$. It seems that the existence of the minimal radius tells us that the nature protect itself from the singularity. In the case of the vacuum solution, the minimal radius is in fact the singular point at zero radius. This proves that the singularity is the consequence of the vacuum solution of the field equations.

In order to theoretically prove that the positive gravitational force could be a source of a dark energy, one can start with the time evolution of the scale factor $\alpha(t)$. This requires the Einstein's field equations together with a way of calculation of density, $\rho(t)$, such as a cosmological equation of state. If the energy-momentum tensor, $T_{\mu \eta}$, is similarly assumed to be isotropic and homogenous, then one obtains the well-known Friedmann equations [27-37, 38]. Following this approach, we obtain the related equations of the universe motion in RAF theory. These equations show that the accelerating expansion of our universe could be driven by the positive gravitational force. This fact theoretically confirms that the positive (repulsive) gravitational force could be the source of the so called dark energy. Of course, this should be confirmed by the related experiments. On the other hand, vacuum solution of the field equations cannot provide any source of the expansion energy. Therefore, this energy has been named as dark energy. This confirms that the dark energy is the consequence of the vacuum solution of the field equations.

This paper is organized as follows. Energy-momentum tensor as function of the field parameters in gravitational field is derived in Sec. II. The theoretical proofs that singularity and dark energy with unknown source are direct consequence of the vacuum solution of the Einstein's field equations are presented in Sec. III. Finally, the related conclusion and 
reference list are presented in Sec. IV and Sec. V, respectively.

\section{ENERGY-MOMENTUM TENSOR AS FUNCTION OF FIELD PARAMETERS IN GRAVITATIONAL FIELD}

In this section, it has been shown the procedure for automatically generation of the energy-momentum tensor as the function of the field parameters in gravitational field. In that sense, we started with the general line element $\mathrm{ds}^{2}$ in an alpha field, given in the first part of RAF theory [7]

$d s^{2}=-\alpha \alpha^{\prime} c^{2} d t^{2}-\kappa\left(\alpha-\alpha^{\prime}\right)_{x} c d t d x-\kappa\left(\alpha-\alpha^{\prime}\right)_{y} c d t d y$ $-\kappa\left(\alpha-\alpha^{\prime}\right)_{z} c d t d z+d x^{2}+d y^{2}+d z^{2}$.

Here $\alpha$ and $\alpha^{\prime}$ are field parameters and $\kappa= \pm 1$. Following the well-known procedure [1-6], this line element can be transformed into the spherical polar coordinates in the nondiagonal form

$$
\begin{aligned}
d s^{2}=- & \alpha \alpha^{\prime} c^{2} d t^{2}-\kappa\left(\alpha-\alpha^{\prime}\right) c d t d r+d r^{2} \\
& +r^{2} d \theta^{2}+r^{2} \sin ^{2} \theta d \phi^{2} .
\end{aligned}
$$

The line element (2) belongs to the well-known form of the Riemann's type line element [10-13]

$$
\begin{aligned}
d s^{2}= & g_{00}\left(d x^{0}\right)^{2}+2 g_{01} d x^{0} d x^{1}+g_{11}\left(d x^{1}\right)^{2} \\
& +g_{22}\left(d x^{2}\right)^{2}+g_{33}\left(d x^{3}\right)^{2} .
\end{aligned}
$$

Here $g_{i j}$ are the components of the related metric tensor. Comparing the equations (2) and (3) we obtain the coordinates and components of the covariant metric tensor, valid for the line element (2):

$$
\begin{aligned}
& d x^{0}=c d t, \quad d x^{1}=d r, \quad d x^{2}=d \theta, \quad d x^{3}=d \phi, \\
& g_{00}=-\alpha \alpha^{\prime}, \quad g_{01}=g_{10}=\frac{-\kappa\left(\alpha-\alpha^{\prime}\right)}{2}, \\
& g_{11}=1, \quad g_{22}=r^{2}, \quad g_{33}=r^{2} \sin ^{2} \theta .
\end{aligned}
$$

Starting with the line element (2) we employ, for the convenient, the following substitutions:

$$
v=\alpha \alpha^{\prime}, \quad \lambda=\kappa\left(\alpha^{\prime}-\alpha\right) / 2 \text {. }
$$

In that case the nondiagonal line element (2) is transformed into the new relation

$$
\begin{aligned}
d s^{2}=- & v c^{2} d t^{2}+2 \lambda c d t d r+d r^{2} \\
& +r^{2} d \theta^{2}+r^{2} \sin ^{2} \theta d \phi^{2} .
\end{aligned}
$$

Using the coordinate system (4), the related covariant metric tensor $g_{\mu \eta}$ of the line element (6) is presented by the matrix form

$$
\left[g_{\mu \eta}\right]=\left[\begin{array}{cccc}
-v & \lambda & 0 & 0 \\
\lambda & 1 & 0 & 0 \\
0 & 0 & r^{2} & 0 \\
0 & 0 & 0 & r^{2} \sin ^{2} \theta
\end{array}\right]
$$

This tensor is symmetric and has six non-zero elements as we expected that should be. The contravariant metric tensor $g^{\mu \eta}$ of the nondiagonal line element (6), is derived by inversion of the covariant one (7)

$$
\left[g^{\mu \eta}\right]=\left[\begin{array}{cccc}
-1 /\left(v+\lambda^{2}\right) & \lambda /\left(v+\lambda^{2}\right) & 0 & 0 \\
\lambda /\left(v+\lambda^{2}\right) & v /\left(v+\lambda^{2}\right) & 0 & 0 \\
0 & 0 & 1 / r^{2} & 0 \\
0 & 0 & 0 & 1 / r^{2} \sin ^{2} \theta
\end{array}\right] .
$$

The determinant of the tensor (7) is given by the relation

$$
\operatorname{det}\left[g_{\mu \eta}\right]=-r^{4}\left(v+\lambda^{2}\right) \sin ^{2} \theta \text {. }
$$

On the other hand, the determinant of the tensor (8) has the form

$$
\operatorname{det}\left[g^{\mu \eta}\right]=-\left(\frac{1}{r^{4}\left(v+\lambda^{2}\right) \sin ^{2} \theta}\right) .
$$

Now we start with the second type of the Christoffel symbols of the metric tensors (7) and (8). These symbols can be calculated by employing the well-known relation [1-6]

$$
\Gamma_{\eta \mu}^{\gamma}=\frac{g^{\kappa \gamma}}{2}\left[g_{\kappa \eta, \mu}+g_{\kappa \mu, \eta}-g_{\eta \mu, \kappa}\right], \kappa, \gamma, \eta, \mu=0,1,2,3 \text {. }
$$

Thus, employing (6), (7), (8) and (11), we obtain the second type Christoffel symbols of the spherically symmetric non-rotating body:

$\Gamma_{00}^{0}=\left(\dot{v}+2 \lambda \dot{\lambda}+v^{\prime} \lambda\right) / D, \Gamma_{01}^{0}=\Gamma_{10}^{0}=v^{\prime} / D, \Gamma_{11}^{0}=-2 \lambda^{\prime} / D$,

$\Gamma_{22}^{0}=-2 \lambda r / D, \Gamma_{33}^{0}=\left(-2 \lambda r \sin ^{2} \theta\right) / D, \Gamma_{00}^{1}=\left(-\dot{v} \lambda+2 v \dot{\lambda}+v v^{\prime}\right) / D$,

$\Gamma_{01}^{1}=\Gamma_{10}^{1}=-v^{\prime} \lambda / D, \Gamma_{11}^{1}=2 \lambda^{\prime} \lambda / D, \Gamma_{22}^{1}=-2 v r / D$

$\Gamma_{33}^{1}=\left(-2 v r \sin ^{2} \theta\right) / D, \Gamma_{12}^{2}=\Gamma_{21}^{2}=\frac{1}{r}, \Gamma_{33}^{2}=-\sin \theta \cos \theta, \Gamma_{13}^{3}=\Gamma_{31}^{3}=\frac{1}{r}$

$\Gamma_{23}^{3}=\Gamma_{32}^{3}=\operatorname{ctg} \theta, \quad D=2\left(v+\lambda^{2}\right), \frac{\partial v}{\partial t}=\dot{v}, \frac{\partial v}{\partial r}=v^{\prime}, \frac{\partial \lambda}{\partial t}=\dot{\lambda}, \frac{\partial \lambda}{\partial r}=\lambda^{\prime}$.

For a static field, the Christoffel symbols $\Gamma_{00}^{0}$ and $\Gamma_{00}^{1}$ are reduced to the simplest forms:

$\Gamma_{00}^{0}=\frac{v^{\prime} \lambda}{2\left(v+\lambda^{2}\right)}, \Gamma_{00}^{1}=\frac{v v^{\prime}}{2\left(v+\lambda^{2}\right)}, \frac{\partial v}{\partial r}=v^{\prime}, \frac{\partial \lambda}{\partial r}=\lambda^{\prime}$.

In the static field, the other Christoffel symbols in (12) are remaining unchanged.

As it is well known, the determinant of the metric tensor of the line element (6) should satisfy the following condition [11-13]

$$
\sqrt{-\operatorname{det}\left[g_{\mu \eta}\right]}=\sqrt{r^{4}\left(\nu+\lambda^{2}\right) \sin ^{2} \theta}=1 \text {. }
$$

Including the normalization of the radius, $r=1$, and the angle $\theta=90^{\circ}$ in (14) we obtain the important relations between the parameters $v$ and $\lambda$ : 


$$
\begin{aligned}
& v+\lambda^{2}=1, v=1-\lambda^{2}, \\
& v^{\prime}=-2 \lambda \lambda^{\prime}, v^{\prime \prime}=-2\left(\lambda^{\prime 2}+\lambda \lambda^{\prime \prime}\right)
\end{aligned}
$$

If we take into account the relations (15), then the Christoffel symbols in (12) and (13) become the functions only of the parameter $\lambda$.

For calculation of the related components of the Riemannian tensor $R_{\beta \eta \mu}^{\kappa}$ and Ricci tensor $R_{\eta \mu}$ of the line element (6) we can employ the following relations [1-6]:

$$
\begin{aligned}
& R_{\beta \eta \mu}^{\kappa}=\Gamma_{\beta \mu, \eta}^{\kappa}-\Gamma_{\beta \eta, \mu}^{\kappa}+\Gamma_{\sigma \eta}^{\kappa} \Gamma_{\beta \mu}^{\sigma}-\Gamma_{\sigma \mu}^{\kappa} \Gamma_{\beta \eta}^{\sigma}, \\
& R_{\eta \mu}=R_{\eta \kappa \mu}^{\kappa}=R_{\mu \eta}, \kappa, \beta, \eta, \mu, \sigma=0,1,2,3 .
\end{aligned}
$$

Applying the Christoffel symbols (12) to the relations (16) we obtain the related Ricci tensor for the static field of the line element (6), with the following components:

$$
\begin{aligned}
& R_{00}=-\left(1-\lambda^{2}\right)\left(\lambda^{\prime 2}+\lambda \lambda^{\prime \prime}+\frac{2 \lambda \lambda^{\prime}}{r}\right), \\
& R_{01}=R_{10}=-\lambda\left(\lambda^{\prime 2}+\lambda \lambda^{\prime \prime}+\frac{2 \lambda \lambda^{\prime}}{r}\right), \\
& R_{11}=\left(\lambda^{\prime 2}+\lambda \lambda^{\prime \prime}+\frac{2 \lambda \lambda^{\prime}}{r}\right), R_{22}=2 \lambda \lambda^{\prime} r+\lambda^{2}, \\
& R_{33}=\left(2 \lambda \lambda^{\prime} r+\lambda^{2}\right) \sin ^{2} \theta .
\end{aligned}
$$

The other components of the Ricci tensor are equal to zero. The related Ricci scalar for the static field is determined by the equation:

$$
\begin{aligned}
& R=g^{\mu \eta} R_{\mu \eta}, \quad \mu, \eta=0,1,2,3, \rightarrow \\
& R=2\left(\lambda^{\prime 2}+\lambda \lambda^{\prime \prime}+\frac{2 \lambda \lambda^{\prime}}{r}\right)+2\left(\frac{2 \lambda \lambda^{\prime}}{r}+\frac{\lambda^{2}}{r^{2}}\right) .
\end{aligned}
$$

In order to calculate the energy-momentum tensor $T_{\mu \eta}$ for the static field, one should employ Ricci tensor (17), Ricci scalar (18) and the Einstein's field equations [1-6] without a cosmological constant $(\Lambda=0)$ :

$$
R_{\mu \eta}-\frac{1}{2} g_{\mu \eta} R=k T_{\mu \eta}, \quad k=\frac{8 \pi G}{c^{4}}, \quad \mu, \eta=0,1,2,3 .
$$

Here $\mathrm{G}$ is the Newton's gravitational constant, $\mathrm{c}$ is the speed of the light in a vacuum and $\mathrm{T}_{\mu \eta}$ is the energy-momentum tensor. Thus, employing of the Einstein's field equations (19) we obtain the following relations for calculation of the components of the energy-momentum tensor $\mathrm{T}_{\mu \mathrm{n}}$ :

$$
\begin{aligned}
& k T_{00}=\left(1-\lambda^{2}\right)\left(\frac{2 \lambda \lambda^{\prime}}{r}+\frac{\lambda^{2}}{r^{2}}\right), k T_{01}=k T_{10}=-\lambda\left(\frac{2 \lambda \lambda^{\prime}}{r}+\frac{\lambda^{2}}{r^{2}}\right), \\
& k T_{11}=(-1)\left(\frac{2 \lambda \lambda^{\prime}}{r}+\frac{\lambda^{2}}{r^{2}}\right), k T_{22}=\left(-r^{2}\right)\left(\lambda^{\prime 2}+\lambda \lambda^{\prime \prime}+\frac{2 \lambda \lambda^{\prime}}{r}\right), \\
& k T_{33}=\left(-r^{2} \sin ^{2} \theta\right)\left(\lambda^{\prime 2}+\lambda \lambda^{\prime \prime}+\frac{2 \lambda \lambda^{\prime}}{r}\right), \quad k=\frac{8 \pi G}{c^{4}} .
\end{aligned}
$$

For calculation of the components of the energy-momentum tensor, $\mathrm{T}_{\mu \eta}$, by the relations (20) we should know the parameter $\lambda$ and its derivations $\lambda^{\prime}$ and $\lambda^{\prime \prime}$ for the related static field. Parameter $\lambda$ is defined by (5) as the function of the field parameters $\alpha$ and $\alpha^{\prime}$

$\lambda=\kappa\left(\alpha^{\prime}-\alpha\right) / 2=-\kappa\left(\alpha-\alpha^{\prime}\right) / 2, \quad \kappa= \pm 1$.

For calculation of the parameter $\lambda$ in a gravitational field we need to know the difference of the field parameters $\left(\alpha-\alpha^{\prime}\right)$, given by the general form in the first part of RAF theory [7]

$\alpha_{1}-\alpha_{1}^{\prime}=\alpha_{3}-\alpha_{3}^{\prime}=2 i \sqrt{\frac{2 U}{m_{0} c^{2}}+\left(\frac{U}{m_{0} c^{2}}\right)^{2}}$.

The second pair is given by the relation

$\alpha_{2}-\alpha_{2}^{\prime}=\alpha_{4}-\alpha_{4}^{\prime}=-2 i \sqrt{\frac{2 U}{m_{0} c^{2}}+\left(\frac{U}{m_{0} c^{2}}\right)^{2}}$.

In the previous relations $U$ and $m_{0}$ are potential energy and rest mass of a particle in a gravitational field, respectively.

Proposition 1. If the gravitational static field is described by the line element (6), then the solution of the Einstein field equations gives the energy momentum tensor $T_{\mu \eta}$ of that field in the following form

$$
\begin{aligned}
T_{\mu \eta}=\left(T_{00}, T_{01}, T_{10}, T_{11}, T_{22}, T_{33}\right) \\
=\left(v,-\lambda,-\lambda,-1, r^{2}, r^{2} \sin ^{2} \theta\right) \frac{(G M)^{2}}{8 \pi G r^{4}} .
\end{aligned}
$$

Here $G$ and $M$ are the gravitational constant and the gravitational mass, respectively.

Proof of the proposition 1. In order to prove of the proposition 1, we should start with the general relations given by (1), (2) to (21). For determination of the field parameters $\alpha$ and $\alpha^{\prime}$ in a gravitational field one need to know the potential energy of the particle in that field. Thus, if a particle with rest mass $m_{0}$ is in a gravitational field, then the potential energy of the particle in that field $U_{g}$ is described by the well-known relation [1-6]

$$
U_{g}=m_{0} V_{g}=m_{0} A_{g 0}=-\frac{m_{0} G M}{r} \text {. }
$$

Here $\mathrm{V}_{\mathrm{g}}=\mathrm{A}_{\mathrm{g} 0}$ is the scalar potential of the gravitational static field, $\mathrm{G}$ is the gravitational constant, $\mathrm{M}$ is a gravitational mass, $r$ is a gravitational radius and $m_{0}$ is a rest mass of the particle that is present in a gravitational static field. For calculation of the difference of the field parameters $\left(\alpha-\alpha^{\prime}\right)$, we can use the general forms given by (22) and (23). Including the substitution $\mathrm{U}=\mathrm{U}_{\mathrm{g}}$ from (25) into (22) and (23) we obtain the difference of the field parameters $\left(\alpha-\alpha^{\prime}\right)$ for a particle in a gravitational static field:

$$
\begin{aligned}
& \alpha_{1}-\alpha_{1}^{\prime}=\alpha_{3}-\alpha_{3}^{\prime}=-2 \sqrt{\frac{2 G M}{r c^{2}}-\left(\frac{G M}{r c^{2}}\right)^{2}}, \\
& \alpha_{2}-\alpha_{2}^{\prime}=\alpha_{4}-\alpha_{4}^{\prime}=2 \sqrt{\frac{2 G M}{r c^{2}}-\left(\frac{G M}{r c^{2}}\right)^{2}} .
\end{aligned}
$$

Applying the results from (26) to the relations in (21) we obtain the two solutions of the parameter $\lambda$ in a gravitational static field 


$$
\lambda=\mp \kappa \sqrt{\frac{2 G M}{r c^{2}}-\left(\frac{G M}{r c^{2}}\right)^{2}} .
$$

Including (27) to (20) we obtain the all items needed for calculations of the components of the energy-momentum tensor $\mathrm{T}_{\mu \eta}$ in a gravitational static field:

$$
\begin{aligned}
& \lambda^{\prime}=\mp \kappa\left[\frac{G M}{r^{2} c^{2}}-\left(\frac{G M}{r^{2} c^{2}}\right)\left(\frac{G M}{r c^{2}}\right)\right] / \sqrt{\frac{2 G M}{r c^{2}}-\left(\frac{G M}{r c^{2}}\right)^{2}}, \\
& \lambda \lambda^{\prime}=\left[-\frac{G M}{r^{2} c^{2}}+\left(\frac{G M}{r^{2} c^{2}}\right)\left(\frac{G M}{r c^{2}}\right)\right], \\
& \frac{2 \lambda \lambda^{\prime}}{r}=2\left[-\frac{G M}{r^{3} c^{2}}+\left(\frac{G M}{r^{2} c^{2}}\right)^{2}\right], \frac{\lambda^{2}}{r^{2}}=\left[\frac{2 G M}{r^{3} c^{2}}-\left(\frac{G M}{r^{2} c^{2}}\right)^{2}\right], \\
& \lambda^{\prime 2}+\lambda \lambda^{\prime \prime}=\left(\lambda \lambda^{\prime}\right)^{\prime}=\frac{2 G M}{r^{3} c^{2}}-3\left(\frac{G M}{r^{2} c^{2}}\right)^{2} .
\end{aligned}
$$

Now, applying the relations (28) to the equations (18) and (20) we obtain the components of the energy-momentum tensor and Ricci scalar valid for the gravitational static field:

$$
\begin{aligned}
& k T_{00}=\left(1-\lambda^{2}\right)\left(\frac{G M}{r^{2} c^{2}}\right)^{2}=v\left(\frac{G M}{r^{2} c^{2}}\right)^{2}, \\
& k T_{01}=k T_{10}=-\lambda\left(\frac{G M}{r^{2} c^{2}}\right)^{2}, k=\frac{8 \pi G}{c^{4}}, \\
& k T_{11}=(-1)\left(\frac{G M}{r^{2} c^{2}}\right)^{2}, \quad k T_{22}=r^{2}\left(\frac{G M}{r^{2} c^{2}}\right)^{2} \text {, } \\
& k T_{33}=\left(r^{2} \sin ^{2} \theta\right)\left(\frac{G M}{r^{2} c^{2}}\right)^{2} \text {, } \\
& R=2\left(\lambda^{\prime 2}+\lambda \lambda^{\prime \prime}+\frac{2 \lambda \lambda^{\prime}}{r}\right)+2\left(\frac{2 \lambda \lambda^{\prime}}{r}+\frac{\lambda^{2}}{r^{2}}\right) \\
& =-2\left(\frac{G M}{r^{2} c^{2}}\right)^{2}+2\left(\frac{G M}{r^{2} c^{2}}\right)^{2}=0 \text {. }
\end{aligned}
$$

From the previous relations we can see that the Ricci scalar is equal to zero. Finally, included parameter $k$ into the relations (29), we obtain the components of the energy-momentum tensor in the gravitational static field

$$
\begin{aligned}
T_{\mu \eta}= & {\left[T_{00}, T_{01}, T_{10}, T_{11}, T_{22}, T_{33}\right] } \\
& =\left[\nu,-\lambda,-\lambda,-1, r^{2}, r^{2} \sin ^{2} \theta\right] \frac{(G M)^{2}}{8 \pi G r^{4}} .
\end{aligned}
$$

Because the relation (30) is equal to the relation (24), the proof of the proposition 1 is finished.

Remarks 1. The previous relations show that the field parameters $(26,27)$ satisfy the full form of the Einstein's field equations with a cosmological constant $\Lambda=0$. In the case of a strong static gravitational field [14-17, 21], the quadratic term
$\left(G M / r c^{2}\right)^{2}$ generates the related energy-momentum tensor $\mathrm{T}_{\mu \eta}$ for the static field. For that case, we do not need to add by hand the related energy-momentum tensor $\mathrm{T}_{\mu \eta}$ on the right side of the Einstein's field equations. In the case of a weak static gravitational field, like in our solar system, we obtain that the quadratic term $\left(G M / r c^{2}\right)^{2} \approx 0$. For that case, the field parameters $(26,27)$ satisfy the Einstein's field equations in a vacuum $\left(\mathrm{T}_{\mu \eta}=0, \Lambda=0\right)$. This corresponds to the well-known Schwarzschild vacuum solution of the line element. But, as we know, the Schwarzschild vacuum solution generates appearances as singularity and dark energy with unknown source. Therefore, it is not allowed to neglect this quadratic term in the weak gravitational field, because in that case we obtain the wrong conclusions about singularity and source of the dark energy. Thus, RAF theory is valid in both weak and strong gravitational fields without singularity and gives the source of dark energy.

The second interpretation of the previous results could be that the quadratic term $\left(G M / r c^{2}\right)^{2}$ generates the cosmological parameter $\Lambda$ as a function of a gravitational radius [18] for $T_{\mu \eta}=0$. It has been shown in [19] that this solution of $\Lambda$ is valid for both Planck's and cosmological scales. Further, the metrics of RAF theory [10] has been applied to the derivation of the generalized relativistic Hamiltonian [20] and dynamic model of nanorobot motion in multipotential field [22].

\section{PROOFS OF THE RAF THEORY PREDICTIONS}

In this section we present the theoretical proofs that no singularity in a gravitational field and that the positive gravitational force could be the source of the dark energy. At the same time, it has been shown that vacuum solution generates singularity in gravitational field and gives no any source of the dark energy.

\section{A. Proof that no a singularity at the Schwarzschild radius}

RAF theory predicts that no a singularity at the Schwarzschild radius. In order to prove this prediction, we calculate the solution of the parameters $v$ and $\lambda$ at the Schwarzschild radius. These parameters are given by (15) and (27) and are valid for the line element (6):

$$
\begin{aligned}
& \lambda=\mp \kappa \sqrt{\frac{2 G M}{r c^{2}}\left(1-\frac{G M}{2 r c^{2}}\right)}, \quad \kappa= \pm 1, \\
& \nu=1-\lambda^{2}=1-\frac{2 G M}{r c^{2}}+\left(\frac{G M}{r c^{2}}\right)^{2}=\left(1-\frac{G M}{r c^{2}}\right)^{2}, \\
& r_{s c h}=\frac{2 G M}{c^{2}}, \rightarrow v_{s c h}=\frac{1}{4}, \lambda_{s c h}=\mp \kappa \sqrt{\frac{3}{4}} .
\end{aligned}
$$

Following the relations in (31) we can see that at the Schwarzschild radius, $r_{s c h}$, parameters $\nu$ and $\lambda$ are regular. Thus, we can say that the metrics of the line element in (6) is regular at the Schwarzschild radius. On that way, the 
theoretical proof of the propositions $\boldsymbol{A}$ (that no a singularity at the Schwarzschild radius) is finished.

In the case of the vacuum solution the quadratic term $\left(G M / r c^{2}\right)^{2}$ does not exists and the relations in (31) are transformed into the new ones:

$$
\begin{aligned}
& \lambda=\mp \kappa \sqrt{\frac{2 G M}{r c^{2}}}, \quad \kappa= \pm 1, \quad v=1-\lambda^{2}=1-\frac{2 G M}{r c^{2}}, \\
& r_{s c h}=\frac{2 G M}{c^{2}}, \quad \rightarrow \quad v_{s c h}=0, \quad \lambda_{s c h}=\mp \kappa, \\
& r<r_{s c h}, v<0, \lambda>\mp \kappa ; r \rightarrow 0, v \rightarrow-\infty, \lambda \rightarrow \mp \infty .
\end{aligned}
$$

From the relations in (31a) we can see that no natural limitation to decreasing of the gravitational radius to the singular point at zero radius. This is theoretical proof that vacuum solution produces singularity in a gravitational field.

\section{B. Proof that there exists a minimal radius at $r=\left(G M / 2 c^{2}\right)$}

RAF theory also predicts that there exists a minimal radius at $r=\left(G M / 2 c^{2}\right)$. This radius prevents singularity at $r=0$, i.e. the nature protects itself.

In order to prove this prediction, we calculate parameters $\nu$ and $\lambda$ in a gravitational static field at the minimal radius:

$$
\begin{aligned}
& \lambda=\mp \kappa \sqrt{\frac{2 G M}{r c^{2}}\left(1-\frac{G M}{2 r c^{2}}\right)}, \quad \kappa= \pm 1, \\
& \nu=1-\lambda^{2}=1-\frac{2 G M}{r c^{2}}+\left(\frac{G M}{r c^{2}}\right)^{2}=\left(1-\frac{G M}{r c^{2}}\right)^{2}, \\
& r_{\text {min }}=\frac{G M}{2 c^{2}}, \rightarrow v=1, \lambda=0, r<r_{\text {min }} \rightarrow \lambda=\lambda_{\text {im }}, \\
& r \rightarrow \infty, v \rightarrow 1, \lambda \rightarrow 0 ; r=\frac{G M}{c^{2}}, \rightarrow v=0, \lambda=1 .
\end{aligned}
$$

From (32), we can see that at the minimal radius parameters $v$ and $\lambda$ are regular and for $r<r_{\min }$ parameter $\lambda$ becomes imaginary number $\lambda=\lambda_{\text {im }}$. Thus, at the minimal radius metrics of the line element (6) is regular. This proves the prediction that there exists a minimal radius at $\mathrm{r}=$ $\left(\mathrm{GM} / 2 \mathrm{c}^{2}\right)$ that prevents singularity at $\mathrm{r}=0$. It seems that the existence of the minimal radius tells us that the nature protect itself from the singularity. On that way, the theoretically proof of the proposition $\boldsymbol{B}$ is finished.

In the case of the vacuum solution the relations in (32) are transformed into the new ones:

$$
\begin{aligned}
& \lambda=\mp \kappa \sqrt{\frac{2 G M}{r c^{2}}}, \quad \kappa= \pm 1, \quad v=1-\lambda^{2}=1-\frac{2 G M}{r c^{2}}, \\
& r_{\text {min }}=\frac{G M}{2 c^{2}}, \quad \rightarrow \quad v=-3, \quad \lambda=\mp 2 \kappa, \\
& r \rightarrow 0, \quad v \rightarrow-\infty, \quad \lambda \rightarrow \mp \infty .
\end{aligned}
$$

From the relations (32a) we can see that the minimal radius in the vacuum solution is in fact the singular point at zero radius.
From the previous consideration, we can see that the metrics of the line element (6) in RAF theory is regular for a gravitational field in the region $r_{\text {min }} \leq r \leq \infty$.

The situation about black holes in the region $r_{\text {min }} \leq r \leq \infty$ of a gravitational field should be considered later. In that sense, it is very interesting to know what Hawking conditionally said about existence of the black holes [24]: "There would be no event horizons and no firewalls. The absence of event horizons means that there are no black holes - in the sense of regimes from which light cannot escape to infinity". The recent investigations of the nature of black holes are also presented in the references $[25,26]$.

\section{Proof that there exists a positive gravitational force}

For the time-invariant (or very slowly changed) alpha field, the gravitational force relations for the gravitational static field are derived in the third part of RAF theory [9]:

$$
\begin{aligned}
& \dot{\lambda}=0, \rightarrow F_{x}=m_{0} \ddot{x}=-\frac{m_{0} G M}{r^{2}}\left(1-\frac{G M}{r c^{2}}\right) \frac{x}{r}, \\
& F_{y}=m_{0} \ddot{y}=-\frac{m_{0} G M}{r^{2}}\left(1-\frac{G M}{r c^{2}}\right) \frac{y}{r}, \\
& F_{z}=m_{0} \ddot{z}=-\frac{m_{0} G M}{r^{2}}\left(1-\frac{G M}{r c^{2}}\right) \frac{z}{r} .
\end{aligned}
$$

The gravitational force relations in (33), generally describe the interactions in the strong fields. But it can also be used in the weak field. As we can see from the relations (33), the gravitational force becomes positive (repulsive) if the term $\left(G M / r c^{2}\right)>1$. This is the case at the extremely strong gravitational fields [14-17, 21]. On the other hand, the gravitational force (33) becomes negative (attractive) in the case where $\left(G M / r c^{2}\right)<1$. This is the case in the relatively weak gravitational field. Thus, in our solar system, the term $\left(G M / r c^{2}\right)$ is too small compare to 1 , and can be neglected. Therefore, our solar system belongs to the relatively weak gravitational field. For an example, on the surface of our Sun the calculation of the amount of this term is $\left(G M / r c^{2}\right) \approx 2.1193 \cdot 10^{-6}<<1$. On the surface of our planet Earth the related influence of the Sun to this term is $\left(G M / r c^{2}\right) \approx 0.989 \cdot 10^{-8}<<1$. Including mass and radius of the planet Earth in this term we obtain that the related gravitational influence of the planet Earth on its surface is $\left(G M / r c^{2}\right) \approx 0.695 \cdot 10^{-9}<<1$. The presented amounts of the term $\left(G M / r c^{2}\right)$ in our solar system could be the answer to the question: why our experience is that gravitational force is only negative (attractive) force?

Further, if the term $\left(G M / r c^{2}\right)=1$, then the gravitational force is equal to zero. This is happened at the gravitational radius $r_{c}=\left(G M / c^{2}\right)$. This radius separates the attractive and repulsive forces in a gravitational field. At the minimal radius, $r_{\min }=\left(G M / 2 c^{2}\right)$ gravitational force is positive (repulsive) $F_{r_{\text {min }}}=\left(4 m_{0} c^{4} / G M\right)$. We can say that the 
nature protects itself from the singularity by producing positive (repulsive) gravitational force $F_{r_{\min }}$ at minimal radius $r_{\min }$.

Generally, we can see that the gravitational force is positive (repulsive) in the region $r_{\min } \leq r<r_{c}$, (see the second part of RAF theory [8] ). At the Schwarzschild radius $r_{s c h}=\left(2 G M / c^{2}\right)$ the gravitational force is negative (attractive) $F_{r_{s c h}}=-\left(m_{0} c^{4} / 8 G M\right)$ and belongs to the negative (attractive) set of gravitation forces in the region $r_{c}<r \leq \infty$.

At the Planck's scale, one can substitute M by Planck's mass $\mathrm{M}_{\mathrm{p}}$ and $\mathrm{r}$ by the Planck's length, $\mathrm{L}_{\mathrm{p}}$. Thus, at the Planck's scale the term $\left(G M_{p} / L_{p} c^{2}\right)=1$. From the relations in (32) we have that $\left(G M_{p} / 2 r_{p m} c^{2}\right)=1$. Thus, the minimal radius of the Planck's mass is $r_{p m}=L_{p} / 2$. The last relation tells us that the Planck's length is in fact minimal diameter of the Planck's mass. Following the relations (33) we can conclude that the gravitational force of the Planck's mass $\mathbf{M}_{\mathrm{p}}$ is positive (repulsive) if the radius $r$ of the Planck's mass is in the region $r_{p m} \leq r<L_{p}$. On the other hand, if the radius $r$ is greater than the Planck's length, $r>L_{p}$, then the gravitational force of the Planck's mass $M_{p}$ is negative (attractive) in the region $L_{p}<r \leq \infty$. For the case where gravitational radius is equal to the Planck's length $\mathrm{L}_{\mathrm{p}}$, $r=L_{p}=2 r_{p m}=r_{p c}$, the gravitational force of the Planck's mass is equal to zero. Here $r_{p c}$ is the radius where the negative acceleration (caused by the Planck's mass) is changes into the positive one and vice versa.

As it is the well known, the related relations for the vacuum solution of gravitational forces have the form [1-6]:

$$
\begin{aligned}
& \dot{\lambda}=0, \rightarrow F_{x}=m_{0} \ddot{x}=-\frac{m_{0} G M}{r^{2}} \frac{x}{r}, \\
& F_{y}=m_{0} \ddot{y}=-\frac{m_{0} G M}{r^{2}} \frac{y}{r}, \quad F_{z}=m_{0} \ddot{z}=-\frac{m_{0} G M}{r^{2}} \frac{z}{r} .
\end{aligned}
$$

From the relations (33a) we can conclude that the gravitational forces can only be negative (attractive) forces. The all experiments in our solar system confirm the relations (33a). But, as it is presented before, our solar system belongs to the relatively weak gravitational field. In such a field the relations (33) are reduced to the vacuum solution form (33a). On the other hand, the vacuum solution (33a) can not be applied to the extremely strong gravitational fields, because it will give incorrect results.

The previous consideration theoretically confirms the prediction of the RAF theory: the gravitational force becomes positive (repulsive) if $\left(\mathrm{GM} / \mathrm{rc}^{2}\right)>1$ that could be the source of the dark energy. Of course, this should be proved by the related experiments. On the other hand, vacuum solution of the field equations tells us nothing about the source of the dark energy.

\section{Proof that a source of dark energy could be a positive gravitational force}

In order to theoretically prove that the positive gravitational force could be a source of a dark energy, one can start with the time evolution of the scale factor $\mathrm{a}(t)$ of the universe expansion. This requires the Einstein's field equations together with a way of calculation of density, $\rho(t)$, such as a cosmological equation of state. If the energy-momentum tensor, $T_{\mu \eta}$, is similarly assumed to be isotropic and homogenous, then the Friedmann equations has the form [27-37, 38]:

$$
\begin{aligned}
& \left(\frac{\dot{\alpha}}{\alpha}\right)^{2}+\frac{\mathcal{K} c^{2}}{\alpha^{2}}-\frac{\Lambda c^{2}}{3}=\frac{8 \pi G}{3} \rho, \\
& 2 \frac{\ddot{\alpha}}{\alpha}+\left(\frac{\dot{\alpha}}{\alpha}\right)^{2}+\frac{\mathcal{K} c^{2}}{\alpha^{2}}-\Lambda c^{2}=-\frac{8 \pi G}{c^{2}} p .
\end{aligned}
$$

Here $\mathbf{\alpha}(t)$ is the scale factor with related time derivations $\dot{\alpha}$ and $\ddot{\alpha}, \mathcal{K}$ is spatial curvature parameter, while $\rho$ and $p$ are fluid mass density and pressure, respectively. The presented equations (34) are the basis of the standard big bang cosmological model including the current $\Lambda$ CDM model. Following the mentioned assumption that the universe is isotropic and homogenous, the model (34) can be used as a first approximation for the evolution of the real, lumpy universe, because it is simple for calculation. Furthermore, the models which calculate the lumpiness in the universe can be added to this model as extensions.

The pair of the equations in (34) is equivalent to the following pair of the equations:

$$
\begin{aligned}
& \frac{\dot{\alpha}^{2}+\mathcal{K} c^{2}}{\alpha^{2}}=\frac{8 \pi G \rho+\Lambda c^{2}}{3}, \\
& \quad \frac{\ddot{\alpha}}{\alpha}=-\frac{4 \pi G}{3}\left(\rho+\frac{3 p}{c^{2}}\right)+\frac{\Lambda c^{2}}{3} .
\end{aligned}
$$

The first equation in (35) has been derived from the 00 component of the Einstein's field equations (19) with $\Lambda \neq 0$. On the other side, the second equation in (35) is derived from the trace of the Einstein's field equations (19) with $\Lambda \neq 0$. It is easy to see that the first equation in (35) can be rewritten into the form of the first equation in (34). The second equation in (35) can be obtained by substitution of the term $(\dot{\alpha} / \alpha)^{2}$ from the first to the second equations in (34). This confirms that the pair of the equations in (34) is equivalent to the related pair of the equations in (35). Some cosmologists call the second equation in (35) as Friedmann acceleration equation and reserve the term Friedmann equation for only the first equation in (35).

Now, one can employ the time derivative of the first equation in (35) and combine it with the second equation in (35). As the result, one obtains the new pair of the equations that are also equivalent to the pairs of the equations in (34) and (35):

$$
\begin{gathered}
\dot{\rho}=-3 \frac{\dot{\alpha}}{\alpha}\left(\rho+\frac{p}{c^{2}}\right), \\
\frac{\ddot{\alpha}}{\alpha}=-\frac{4 \pi G}{3}\left(\rho+\frac{3 p}{c^{2}}\right)+\frac{\Lambda c^{2}}{3} .
\end{gathered}
$$


Here the spacial curvature index serving as a constant of the integration for the second equation in (36). The first equation in (36) can be derived also from thermo-dynamical consideration and is equivalent to the first law of thermodynamics, assuming that the expansion of the universe is an adiabatic process. This assumption has been implicitly included into the derivation of the Friedmann - Lemaitre Robertson - Walker metric. The second equation in (36) states that both the energy density and the pressure cause the expansion rate of the universe, $\dot{\alpha}$, to decrease. It means that both the energy density and the pressure cause a deceleration in the expansion of the universe. This is the consequence of gravitation, including that pressure is playing a similar role to that of energy (or mass) density. This is, of course, in accordance with the principles of general relativity. On the other side, the cosmological constant, $\Lambda$, causes acceleration in the expansion of the universe.

Further, one can introduce the density parameter, $\Omega$, as the ratio of the actual (or observed) density, $\rho$, to the critical

density, $\rho_{c}$, of the Friedmann universe. This ratio determines the overall geometry of the universe. It is well known that in the earlier models, which did not include a cosmological constant term, the critical density was regarded also as the watershed between an expanding and contracting Universe. Recently, the critical density is estimated to be approximately five atoms (of monatomic hydrogen) per cubic meter, whereas the average density of ordinary matter in the Universe is believed to be 0.2 atoms per cubic meter [37]. Meanwhile, a much greater density comes from the unidentified dark matter. From the General Relativity, we know that both ordinary and dark matter contribute in favor of contracting of the universe. But, the largest part of density comes from the so-called dark energy that accounts for the cosmological constant term. Although the total density of the universe is equal to the critical density (exactly, up to measurement error), the dark energy does not lead to contraction of the universe. In fact, the dark energy contributes in favor of expanding of the universe.

Recently, the spatial geometry of the universe has been measured by the WMAP spacecraft. The result of that measuring showed that the spatial geometry of the universe is nearly flat. Following this result, one can conclude that the universe can be well approximated by a model without the spatial curvature parameter $\mathcal{K}$ (i.e. $\mathcal{K}=0$ ). Meanwhile, this does not necessarily imply that the universe is infinite. It is because our measuring is related to the observation part of the universe that is limited, but the universe is much larger than the part we can see.

Further we show a new approach to the description of the universe motion. This approach is based on the new Relativistic Alpha Field (RAF) theory [7,8,9]. It has been shown that the general nondiagonal form of the line element, $\mathrm{ds}^{2}$, of RAF theory, in the spherical polar coordinates, can be described by the equation [7]:

$$
\begin{aligned}
& d s^{2}=-\alpha \alpha^{\prime} c^{2} d t^{2}-k\left(\alpha-\alpha^{\prime}\right) c d t d r \\
& +d r^{2}+r^{2} d \theta^{2}+r^{2} \sin ^{2} \theta d \phi^{2}, \quad v=\alpha \alpha^{\prime}, \lambda=k\left(\alpha^{\prime}-\alpha\right) / 2, \\
& d s^{2}=-v c^{2} d t^{2}+2 \lambda c d t d r+d r^{2}+r^{2} d \theta^{2}+r^{2} \sin ^{2} \theta d \phi^{2} .
\end{aligned}
$$

Here, $\boldsymbol{c}$ is the speed of the light in a vacuum, $\boldsymbol{r}$ is a radius vector, $\boldsymbol{\theta}$ is an angle between radius vector $\boldsymbol{r}$ and $\boldsymbol{z}$-axis, and $\boldsymbol{\phi}$ is an angle between projection of a radius vector $\boldsymbol{r}$ on $(x-y)$ plane and $x$-axis. Parameter $\mathrm{k}= \pm 1$ (see [7]). The general solutions of that line element are presented by the relations:

$$
\begin{gathered}
\Lambda=\text { const. } \rightarrow \quad v=1-\frac{2 G M}{r c^{2}}-\frac{\Lambda}{3} r^{2}, \lambda=\sqrt{\frac{2 G M}{r c^{2}}+\frac{\Lambda}{3} r^{2}}, \\
\alpha_{1}=1-\sqrt{\frac{2 G M}{r c^{2}}+\frac{\Lambda}{3} r^{2}}, \alpha_{1}^{\prime}=1+\sqrt{\frac{2 G M}{r c^{2}}+\frac{\Lambda}{3} r^{2}}, \\
R A F T, \rightarrow v=1-\frac{2 G M}{r c^{2}}+\left(\frac{G M}{r c^{2}}\right)^{2}, \lambda=\sqrt{\frac{2 G M}{r c^{2}}-\left(\frac{G M}{r c^{2}}\right)^{2}}, \\
\alpha_{1}=1-\sqrt{\frac{2 G M}{r c^{2}}-\left(\frac{G M}{r c^{2}}\right)^{2}}, \quad \alpha_{1}^{\prime}=1+\sqrt{\frac{2 G M}{r c^{2}}-\left(\frac{G M}{r c^{2}}\right)^{2}} .
\end{gathered}
$$

Here RAFT means RAF theory, GM $/ \mathrm{c}^{2}$ is the Newton's constant of integration, $\mathrm{G}$ is a gravitational constant, $\mathrm{M}$ is a total gravitational mass, $r$ is a gravitational radius, $\Lambda$ is a cosmological constant and $\mathrm{c}$ is the speed of the light in a vacuum. In RAF theory, it is assumed that the cosmological constant $\Lambda=0$ and the energy momentum tensor $T_{\mu \eta} \neq 0$. If displacement four-vector $\mathrm{dX}$ is defined in frame $\mathrm{K}$ by the expression:

$d X \rightarrow K(c d t, d r, d \theta, d \phi)=\left\{d x^{i}\right\}, i=0,1,2,3$,

then the related covariant metric tensor of the line element (37) has the form $[7,8,9]$ :

$$
\left[g_{\mu \eta}\right]=\left[\begin{array}{cccc}
-v & \lambda & 0 & 0 \\
\lambda & 1 & 0 & 0 \\
0 & 0 & r^{2} & 0 \\
0 & 0 & 0 & r^{2} \sin ^{2} \theta
\end{array}\right] .
$$

Here the non-null components of the metric tensor $g_{\mu \eta}$ are given by the relations:

$$
\begin{aligned}
& \Lambda=\text { const. } \rightarrow g_{00}=-v=-\left(1-\frac{2 G M}{r c^{2}}-\frac{\Lambda}{3} r^{2}\right), \\
& g_{01}=g_{10}=\lambda=\sqrt{\frac{2 G M}{r c^{2}}+\frac{\Lambda}{3} r^{2}}, g_{11}=1, g_{22}=r^{2}, g_{33}=r^{2} \sin ^{2} \theta . \\
& R A F T \rightarrow g_{00}=-v=-\left(1-\frac{2 G M}{r c^{2}}+\left(\frac{G M}{r c^{2}}\right)^{2}\right), \\
& g_{01}=g_{10}=\lambda=\sqrt{\frac{2 G M}{r c^{2}}-\left(\frac{G M}{r c^{2}}\right)^{2}}, g_{11}=1, g_{22}=r^{2}, g_{33}=r^{2} \sin ^{2} \theta .
\end{aligned}
$$

The related determinant of the metric tensor (40) has the forms:

$$
\begin{gathered}
\operatorname{det}\left[g_{\mu \eta}\right]=-r^{4}\left(v+\lambda^{2}\right) \sin ^{2} \theta, r=1, \theta=\frac{\pi}{2}, \rightarrow \\
\operatorname{det}\left[g_{\mu \eta}\right]=-\left(v+\lambda^{2}\right)=-1, \quad v=1-\lambda^{2} .
\end{gathered}
$$


In the previous relation we use the normalization for $r=1, \quad \theta$ $=\pi / 2$, and the well-known condition for the metric tensor of the line element $\operatorname{det}\left(\mathrm{g}_{\mu \eta}\right)=-1$. As the result, we obtain the simple relation between field parameters $v$ and $\lambda$.

If the line element in an alpha field is defined by the relations (37) to (42) then the dynamic model of the Universe motion for $\Lambda=$ const. is given by the equations [38]:

$$
\begin{aligned}
\Lambda=\text { const. } \rightarrow\left(\frac{\dot{r}}{r}\right)^{2} & +\frac{\mathcal{K} c^{2}}{r^{2}}-\frac{\Lambda c^{2}}{3}=\frac{8 \pi G}{3} \rho, \\
& \ddot{r}=-\frac{4 \pi G}{3}\left(\rho+\frac{3 p}{c^{2}}\right)+\frac{\Lambda c^{2}}{3} .
\end{aligned}
$$

Comparing the first equation in (43) with the first Friedmann equation in (34), and the second equation in (43) with the second Friedmann equation in (35), one can conclude that both equations in (43) have the same forms as the Friedmann equations if we include the substitutions $r=\alpha, \dot{r}=\dot{\alpha}$ and $\ddot{r}=\ddot{\alpha}$. Thus, if the equations (43) describe the universe motion, then the radial coordinate $r(\mathrm{t})$ has the roll of the scale factor $\alpha(\mathrm{t})$.

The second equation in (43) states that both the energy density and the pressure cause a deceleration in the expansion of the universe. This is the consequence of gravitation, including that pressure is playing a similar role to that of energy (or mass) density. This is, of course, in accordance with the principles of general relativity. On the other side, the cosmological constant, $\Lambda$, causes acceleration in the expansion of the universe. In that case the cosmological constant, $\Lambda$, plays the role of the dark energy.

If the line element in an alpha field is defined by the relations (37) to (42) then the dynamic model of the Universe motion in RAF theory is given by the equations [38]:

$$
\begin{array}{r}
R A F T \rightarrow\left(\frac{\dot{r}}{r}\right)^{2}+\frac{\mathcal{K} c^{2}}{r^{2}}=\frac{8 \pi G}{3} \rho\left(1-\frac{2 \pi G}{3} \frac{\rho r^{2}}{c^{2}}\right), \\
\frac{\ddot{r}}{r}=-\frac{4 \pi G}{3}\left(\rho+\frac{3 p}{c^{2}}\right)\left(1-\frac{4 \pi G}{3} \frac{\rho r^{2}}{c^{2}}\right) .
\end{array}
$$

Comparing the first equation in (44) with the first Friedmann equation in (34), one can conclude that both equations have the same forms of the two parts of the left side and the first part of the right side if the substitutions $r=\alpha$ and $\dot{r}=\dot{\alpha}$ are valid.

Comparing the second equation in (44) with the second Friedmann equation in (35), one can conclude that both equations have the same forms of the left side and of the first part of the right side if the substitutions $r=\alpha$ and $\ddot{r}=\ddot{\alpha}$ are valid. Thus, if the equations (44) describe the universe motion, then the radial coordinate $r(\mathrm{t})$ has the roll of the scale factor $\alpha(t)$. The first part of the right side of the second equation in (44) states that both the energy density and the pressure cause a deceleration in the expansion of the universe. This is the consequence of gravitation, including that pressure is playing a similar role to that of energy (or mass) density. This is, of course, in accordance with the principles of general relativity. On the other hand, the second part of the right side of the second equation in (44) causes acceleration in the expansion of the universe. This is the consequence of the positive (repulsive) gravitation force presented by RAF theory in $[7,8,9]$.

This fact theoretically confirms that the positive (repulsive) gravitational force could be the source of the so called dark energy. Of course, this should be confirmed by the related experiments.

\section{CONCLUSION}

In this paper, we show that the new Relativistic Alpha Field Theory (RAFT) proposes solution of the field equations without appearance of singularity and offers the source of dark energy. On that way RAF theory extends the application of GRT to the extremely strong fields at the Planck's scale. This conclusion is based on the theoretically proofs that: a) no a singularity at the Schwarzschild radius, b) there exists a minimal radius at $r=\left(\mathrm{GM} / 2 \mathrm{c}^{2}\right)$ that prevents singularity at $\mathrm{r}=$ 0 , i.e. the nature protects itself and d) a positive (repulsive) gravitational force could be the source of dark energy. On that way, we theoretically proved that the singularity and dark energy are consequences of the vacuum solution of Einstein's field equations.

\section{ACKNOWLEDGMENTS}

The author wishes to thank to the anonymous reviewers for a variety of helpful comments and suggestions. This work is supported by grants (120-1201842-3048) from the National Scientific Foundation of Republic of Croatia.

\section{REFERENCES}

[1] A. Einstein, Ann. Phys. 49, 769-822 (1916).

[2] A. Einstein, The Meaning of Relativity (Princeton Univ. Press, Princeton, 1955).

[3] C. Sean, Spacetime and Geometry: An introduction to General Relativiry (Amazon.com Books.htm, Hardcover, 2003).

[4] S. Weinberg, Gravitation and Cosmology: Principles and Application of the General Theory of Relativity (Gebundene Ausgabe, RelEspWeinberg.pdf., 1972).

[5] S. W. Hawking, G. F. R. Ellis, The Large Scale Structure of Space-Time (Univ. Press, Cambridge, 1973).

[6] M. Blau, Lecture Notes on General Relativity (A. Einstein Center for Fundamental Physics, Univ. Bern, Bern, 2012, 2014).

[7] B. M. Novakovic, Relativistic alpha field theory - Part I: Determination of Field Parameters, International Journal of New Technology and Research (IJNTR) ISSN:2454-4116, Volume-1, Issue-5, September 2015, Pages 23-30.

[8] B. M. Novakovic, Relativistic alpha field theory - Part II: Does a Gravitational Field Could be Without Singularity? International Journal of New Technology and Research (IJNTR) ISSN:2454-4116, Volume-1, Issue-5, September 2015, Pages 31-38.

[9] B. M. Novakovic, Relativistic alpha field theory - Part III: Does Gravitational Force Becomes Positive if $(\mathrm{GM} / \mathrm{rc} 2)>1$ ? International Journal of New Technology and Research (IJNTR) ISSN:2454-4116, Volume-1, Issue-5, September 2015 Pages 39-47.

[10] B. M. Novakovic, Int. J. of Comput. Anticip. Syst., IJCAS 27, p. 93 (2014).

[11] S. Gallot, D. Hullin, and D. J. Lafontane, Riemannian Geometry ( Springer-Verlag, Berlin, New York, ed. 3, 2004).

[12] C. T. J. Dodson, and T. Poston, Tensor Geometry. Graduate Texts in Mathematics (Springer-Verlag, Berlin, New York, ed. 2, 1991), p. 130

[13] M. T. Vaughin, Introduction to Mathematical Physics (Wiley-VCH Verlag GmbH \& Co., Weinheim, 2007).

[14] D. H. Perkins, Introduction to High Energy Physics (Cambridge Univ. Press, Cambridge, 2000).

[15] D. Sherman, et al., Nat. Phys. 11, 1-5 (2015).

[16] J. Steinhauer, Nat. Phys. 10, 864-869 (2014).

[17] M. Meckel, et al. Nat. Phys. 10, 594 - 600 (2014). 
[18] B. M. Novakovic, D. Novakovic, and A. Novakovic. in Proceedings of the Sixth Int. Conf. on Comp. Anticip. Syst., Liege, 2003, edited by D. Dubois (University of Liege, Liege 2003), AIP-CP 718, p.133 (2004). DOI: $10.1063 / 1.1787318$

[19] B. M. Novakovic, D. Novakovic, and A. Novakovic, in Proceedings of the Seventh Int. Conf. on Comp. Anticip. Syst., Liege, 2005, edited by D. Dubois (University of Liege, Liege 2005), AIP-CP 839, p.144, (2006). DOI: 10.1063/1.2216624.

[20] B. M. Novakovic, in Proceedings of the Ninth Int. Conf. on Comp. Anticip. Syst., Liege, 2009, edited by D. Dubois (University of Liege, Liege 2009), AIP-CP 1303, p. 141 (2010). DOI: 10.1063/1.3527149.

[21] R. Ding, et al. Phys. Rev. D 92, (015008) (2015).

[22] B. M. Novakovic, Strojarstvo 53 (2), 103-111 (2011).

[23] M. Rees, Just Six Numbers, (Orion Book, London, p. 81, p. 82., 2000).

[24] S. W. Hawking: "There no Black Holes" in Information Preservation and Weather Forecasting for Black Holes, arXiv: 1401.5761 [hep-th], 22 Jan. 2014

[25] A. Almheiri, D. Marolf, J. Polchinski, J. Sully, Black Holes: Complementarity or Firewalls?, J. High Energy Phys. 2, 062 (2013).

[26] D. Gerosa, E. Berti, Are merging black holes born from stellar collapse or previous mergers?, Phys. Rev. D 95, 124096 (2017), 26 June 2017

[27] A. Friedmann, Über die Krümmung des Raumes. Z.Phys. 10 (1), pp. 377-386 (in German), (1922). A. Friedman, On the Curvature of Space. General Relativity and Gravitation 31 (12), pp. 1991-2000 (in English), (1999).

[28] A. Friedmann, Über die Möglichkeit einer Welt mit konstanter negativer Krümmung des Raumes. Z.Phys. 21 (1), pp. 326-332 (in German), (1924). A. Friedman, On the Possibility of a World with Constant Negative Curvature of Space. General Relativity and Gravitation 31 (12), pp. 2001-2008 (in English), (1999).

[29] G.Lemaitre, Expansion of the Universe, a Homogeneous Universe of Constant Mass and Increasing Radius Accounting for the Radial Velocity of Extra Galactic Nebulae. Monthly Notices of the Royal Astronomical Society 91, pp. 483-490, (1931).

[30] G. Lemaître, L'Univers en Expansion. Annales de la Société Scientifique de Bruxelles A53, pp. 51-85 (in French), (1933).

[31] H.P. Robertson, Kinematics and World Structure. Astrophysical Journal 82, pp. 284-301, (1935).

[32] H.P. Robertson, Kinematics and World Structure II. Astrophysical Journal 83, pp. 187-201, (1936).

[33] H.P. Robertson, Kinematics and World Structure III. Astrophysical Journal 83, pp. 257-271, (1936).

[34] A. G. Walker, On Milne's Theory of World Structure. Proceedings of the London Mathematical Society 242 (1), pp. 90-127, (1937)

[35] L. Bergström, A. Goobar, Cosmology and Particle Astrophysics. Sprint, p. 61, 2nd ed., ISBN 3540329242, (2006).

[36] P. Ojeda, H. Rosu, Supersymmetry of FRW Barotropic Cosmologies, International Journal of Theoretical Physics 45 (6), pp. 1191-1196, (2006).

[37] Wikipedia, the free encyclopedia. (2012). FLRW Metrics. http://en.wikipedia.org/wiki/Friedmann_Lemaitre_Robertson_Walker _Metrics, Retrieved 19 March (2012)

[38] B. M. Novakovic, Is Positive Gravitational Force Source of Dark Energy?, International Journal of New Technology and Research (IJNTR) ISSN:2454-4116, Volume-1, Issue-7, November 2015, Pages 06-13.

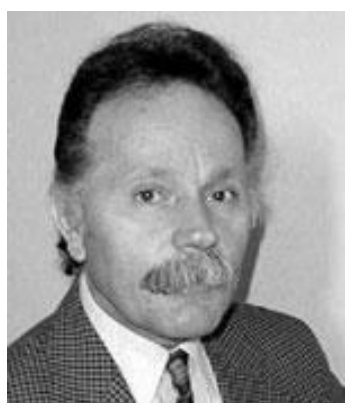

Branko Novakovic is a Professor emeritus at FSB - University of Zagreb, Croatia. Prof. Novakovic received his $\mathrm{PhD}$ from the University of Zagreb in 1978. His research of interest includes physics, control systems, robotics, neural networks, and fuzzy control. $\mathrm{He}$ is author of three books, Relativistic Alpha Field Theory (RAFT, e-book, 2016), Control Methods in Robotics, Flexible Manufacturing Systems and Processes (1990) and Control Systems (1985), He is also co-author of a book Artificial Neural Networks (1998). He has published over 230 research papers in his research of interest. 